\title{
Individual and Total Sugar Contents of 83 Malaysian Foods
}

\author{
Norhayati Mustafa Khalid ${ }^{1}$, Mohd Fairulnizal Md Noh ${ }^{1}$, Mohd Naeem Mohd Nawi ${ }^{1}$, Nazline Miasin Kehid ${ }^{1}$, \\ Aswir Abd Rashed ${ }^{1}$, Wan Sulong Wan Omar $^{2}$, Norliza Abd Hamid ${ }^{2}$, Janarthini Subramaniam² \& Rusidah \\ Selamat ${ }^{3}$
}

${ }^{1}$ Nutrition Unit, Cardiovascular, Diabetes and Nutrition Research Centre, Institute for Medical Research, Jalan Pahang, 50588 Kuala Lumpur, Malaysia

${ }^{2}$ Food Section, Chemistry Department, Environmental Health Division, Chemistry Department, Jalan Sultan, 46661 Petaling Jaya, Malaysia

${ }^{3}$ Food Division, Aras 1, Blok E3, Parcel E, Pusat Pentadbiran Kerajaan Persekutuan, 62590 Putrajaya, Malaysia

Correspondence: Norhayati Mustafa Khalid, Nutrition Unit, Cardiovascular, Diabetes and Nutrition Research Centre, Institute for Medical Research, Jalan Pahang, 50588 Kuala Lumpur, Malaysia. Tel: 60-326-162-511. E-mail: norhayati@imr.gov

Received: January 19, 2018 Accepted: February 9, 2018 Online Published: April 3, 2018

doi:10.5539/jfr.v7n3p58 URL: https://doi.org/10.5539/jfr.v7n3p58

\begin{abstract}
As part of the effort in updating and expanding the carbohydrate data in Malaysian Food Composition Databases, 83 foods were selected based on the most commonly consumed foods and food products by Malaysian. The samples include 31 cereal products, 9 starchy roots and tubers products, 4 legume products, 11 nut and seed products, 4 vegetables, 5 fruits, 15 sugar and syrup products, 2 meat products and 2 oil and fat products. Individual sugars (fructose, glucose, sucrose, lactose and maltose) were analysed using High Performance Liquid Chromatography with Refractive Index Detector. Most of the cereal products contained sucrose, glucose, fructose, lactose and maltose. Four starchy root and tuber products contained sucrose, glucose and fructose. Sucrose was detected in all legume, nut and seed products. Most vegetables contained fructose while all fruits contained glucose and fructose. In addition, all syrups contained sucrose except for kiwi and lime cordial. Overall, sugar and syrup products contained the highest total sugar content (15.00-65.52 g/ 100g) while vegetables were the lowest for total sugar content $(2.74-4.83 \mathrm{~g} / 100 \mathrm{~g})$.
\end{abstract}

Keywords: individual sugars, total sugar, Malaysian, foods

\section{Introduction}

A comprehensive Malaysian Food Composition Table in Malaysia was published in 1988 and has been revised in 1997 (Siong, Noor, Azudin, \& Idris, 1997). However, carbohydrate was determined by difference and reported for all foods. As part of the continuous efforts to update and expand the carbohydrate data in its database, 83 Malaysian foods were selected based on most commonly consumed foods and measured for its individual and total sugar content.

Sugars were defined as the sum of all free mono and disaccharides which would include glucose, fructose, galactose, lactose, sucrose and maltose (Kahn \& Sievenpiper, 2014). Glucose and fructose are naturally occurring monosaccharides present in fruit and honey while sucrose is a disaccharide extracted from sugar cane and beet (Tappy \& Le, 2010). Maltose is a disaccharide formed by two units of glucose. Combination of galactose and glucose formed lactose, a disaccharide normally found in milk. Data from the 2003 Malaysian Adult Survey (MANS) showed that Malaysian adults consumed 30 grams of sweetened condensed milk (equivalent to 16 grams sugar) and 21 grams of table sugar per day (MOH, 2008). Increased intake of foods high in sugar content and excessive caloric intake is associated with the major factor contributing to the rise of obesity and chronic disease (Bray, 2013; Morenga, Mallard, \& Mann, 2013).

With the increase prevalence of diet-related chronic diseases such as diabetes, the information on sugar content in foods are needed to increase the effectiveness of nutritional advices. Recognizing the importance of sugar in the aetiology and prevention of diseases in the country, there is a need to update the Malaysian Food Composition Database with individual and total sugar data which were not included in the 1997 Malaysian Food Composition Database. 


\section{Materials and Methods}

\subsection{Selection of Foods}

The choice of foods to be analysed was based on the data provided from Malaysian Adult Nutrition Survey carried out between October 2002 and December 2003. The most consumed foods, taken from both the 24-hour diet recall and food frequency questionnaire methods, were selected for analysis. These foods were divided into several food categories. Foods included 31 cereal products, 9 starchy root and tuber products, 4 legume products, 11 nut and seed products, 4 vegetables, 5 fruits, 15 sugar and syrup products, 2 meat products and 2 oil and fat products.

\subsection{Sample Collection}

A stratified sampling plan based on the Protocol for Sampling and Methods of Analysis for Malaysian Food Composition (2011) was used for sample collection. Foods were sampled from local supermarkets in the Klang Valley. For most of the foods (cereal products, starchy root and tuber products, legume products, nut and seed products, sugar and syrup products and meat products), six top national brands were purchased. In the cases where only one brand dominated, then only that brand was used. The fresh vegetables and fruits were purchased from three major grocery chains (supermarket, wet market and Pasar Tani).

\subsection{Sample Preparation}

Cereal products, starchy root and tuber products, legume products, nut and seed products and meat products were grounded into fine powder using a food grinder, transferred into air tight containers and kept at ambient temperature until further analysis. While for vegetables and fruits, the samples were washed with tap water to remove impurities and non-edible parts such as skin and seed were removed. Then, the edible part of vegetables and fruits were grounded using food processor into homogenous form. Samples were stored in air tight containers and kept at $4^{\circ} \mathrm{C}$. Analyses were carried out in duplicate.

\subsection{Sugar Analysis}

The analysis for mono and disaccharides (fructose, glucose, sucrose, lactose and maltose) was carried out using High Performance Liquid Chromatography (HPLC) with Refractive Index Detector (Waters, USA). One gram of sample was weighed into a $10 \mathrm{ml}$ centrifuge tube and then dissolved in $25 \mathrm{ml}$ acetonitrile:deionized water (50:50 v:v). This solution was then centrifuged for 30 minutes at $3200 \mathrm{rpm}$. The supernatant was filtered using 0.45 nylon filter into an HPLC vial. Separation were carried out on an amino-bonded column with a mobile phase of acetonitrile:deionized water:triethylamine (75:25:0.2) and sugar content were determined by Refractive Index Detector against the standard solution. Standard solutions of $0.1,0.2,0.5,0.8$ and $1.0 \mathrm{ppm}$ were prepared and analysed prior to samples analysis. As an effort to ensure the reliability of the data, quality control samples were used and analysed simultenously after calibration of the standards. This was done prior to samples analysis.

Biscuits and cordial were used as quality control for solid and liquid samples. The result for control was acceptable if its fall within the two standard deviation of the mean previously set as acceptable limit for the control.

\subsection{Statistical Analysis}

All data were calculated using Excel 2010 and results were reported as mean and standard deviation.

\section{Results and Discussion}

Table 1 shows the individual and total sugar content of some Malaysian foods. Results for cereal products showed various levels of sucrose, glucose, fructose, lactose and maltose. Total sugar content varied form $0.00 \mathrm{~g}$ $/ 100 \mathrm{~g}$ for rice and $24.56 \pm 6.48 \mathrm{~g} / 100 \mathrm{~g}$ for raisin biscuit. Sucrose is the major contributing sugar to the total sugar content in selected commercial biscuits in Malaysia (Norhayati et al., 2015). For starchy root and tuber products, only four foods contained sucrose, glucose and fructose and total sugar content varied between $0.00 \mathrm{~g}$ $/ 100 \mathrm{~g}$ for sago flour up to12.54 \pm 8.39 for spicy tapioca chips. For legume products, all four foods contained sucrose. Total sugar content varied between $2.15 \pm 0.50 \mathrm{~g} / 100 \mathrm{~g}$ for carob flour up to $42.23 \pm 15.85 \mathrm{~g} / 100 \mathrm{~g}$ for sweet soy sauce. All eleven nuts and seeds products contained sucrose and only three contained fructose and glucose. None of the nuts and seeds products contained lactose and total sugar content varied between $1.10 \pm 0.05$ $\mathrm{g} / 100 \mathrm{~g}$ for pumpkin seed and $40.97 \pm 8.41 \mathrm{~g} / 100 \mathrm{~g}$ for crush peanut. All vegetables were found to contain fructose except yellow capsicum which found to contain glucose. Total sugar content for vegetables varied between $2.74 \pm 0.64 \mathrm{~g} / 100 \mathrm{~g}$ for yellow capsicum and $4.83 \pm 1.30 \mathrm{~g} / 100 \mathrm{~g}$ for red capsicum. All five fruits contained glucose and fructose and total sugar content varied between $2.92 \pm 0.59 \mathrm{~g} / 100 \mathrm{~g}$ for honeydew and $10.70 \mathrm{~g} / 100 \mathrm{~g}$ for pomegranate. All fifteen sugar and syrup products contained sucrose except for kiwi and lime cordial and all cordial contained glucose and fructose. Total sugar content for sugar and syrup products varied 
between $15.00 \pm 5.52 \mathrm{~g} / 100 \mathrm{~g}$ for root beer cordial and $65.52 \pm 13.53 \mathrm{~g} / 100 \mathrm{~g}$ for pineapple cordial. For meat products, beef sausage contained maltose meanwhile chicken sausage contained fructose, glucose and maltose. Total sugar content for meat products varied from $0.255 \mathrm{~g} / 100 \mathrm{~g}$ to $0.543 \mathrm{~g} / 100 \mathrm{~g}$. Oils and fats products contained sucrose, glucose and fructose and total sugar content varied between $8.59 \pm 1.20 \mathrm{~g} / 100 \mathrm{~g}$ for mayonnaise and $15.47 \pm 2.40 \mathrm{~g} / 100 \mathrm{~g}$ for thousand island sauce. The total sugar values include free monosaccharide and disaccharide intrinsic to the plant and any added sugar during food preparation and manufacture (Sanchez-Castillo et al., 2000). Sugars and syrups products content the highest total sugar compared to other food categories because the main ingredient for these products is sugar as stated at the ingredient list. Added sugar refers to sugars and syrups added to foods during processing or preparation and sugar and syrups added at the table (Van Horn, Johnson, Flickinger, Vafiadis, \& Yin-Piazza, 2010). The types of added sugars frequently used in food supply are fructose and non-fructose rich corn syrups, cane and beet sugar, honey and other edible syrups. Usually, these sugars are added as ingredients in processed foods, cereal and bakery products, beverages, dairy products, candy and other confectionary items (Sigman-Grant \& Morita 2003).

Table 1. Individual and Total Sugars content of 83 Malaysian Foods ( $\mathrm{g} / 100 \mathrm{~g}$ as eaten)

\begin{tabular}{|c|c|c|c|c|c|c|c|c|}
\hline English name & Malaysian name & $\mathrm{n}$ & Sucrose & Glucose & Fructose & Lactose & Maltose & Total Suga \\
\hline \multicolumn{9}{|l|}{ Cereal products } \\
\hline Rice, boil in beg rice & Ketupat & 6 & - & - & - & - & - & - \\
\hline Rice, siam & Beras siam & 6 & - & - & - & - & - & - \\
\hline Rice, basmathi & Beras basmathi & 6 & - & - & - & - & - & - \\
\hline Rice, fragrant & Beras wangi & 6 & - & - & - & - & - & - \\
\hline Biscuit, chocolate chip & Biskut coklat cip & 6 & $24.17 \pm 4.67$ & - & - & - & - & $24.17 \pm 4.67$ \\
\hline Wafer, chocolate, full coated & Wafer salut coklat & 6 & $32.45 \pm 3.95$ & $0.83 \pm 2.04$ & - & $5.85 \pm 3.73$ & - & $39.13 \pm 4.14$ \\
\hline Biscuit, corn & Biskut jagung & 6 & $8.80 \pm 6.19$ & $2.15 \pm 3.33$ & $2.18 \pm 3.38$ & $0.48 \pm 1.18$ & $0.40 \pm 0.98$ & $14.02 \pm 3.21$ \\
\hline Biscuit, cracker with sugar & Biskut kraker bergula & 6 & $12.28 \pm 2.85$ & - & - & - & $2.63 \pm 0.38$ & $14.92 \pm 2.95$ \\
\hline $\begin{array}{l}\text { Biscuit, crackers, } \\
\text { vegetable flavor }\end{array}$ & $\begin{array}{l}\text { Biskut kraker } \\
\text { perisa sayuran }\end{array}$ & 6 & $7.45 \pm 5.91$ & - & - & $0.85 \pm 1.36$ & $1.42 \pm 1.63$ & $9.72 \pm 5.69$ \\
\hline Biscuit, cream filled & Biskut berkrim & 6 & $23.25 \pm 5.87$ & - & - & $1.60 \pm 1.75$ & $0.80 \pm 1.25$ & $25.65 \pm 4.91$ \\
\hline Biscuit, oatmeal & Biskut oat & 6 & $15.67 \pm 2.12$ & - & - & $0.38 \pm 0.94$ & $0.38 \pm 0.94$ & $16.43 \pm 3.06$ \\
\hline Biscuit, shortbread & Biskut shortbread & 6 & $16.38 \pm 8.79$ & $1.05 \pm 2.57$ & - & - & $0.37 \pm 0.90$ & $17.80 \pm 5.78$ \\
\hline $\begin{array}{l}\text { Biscuit, whole } \\
\text { meal crackers }\end{array}$ & $\begin{array}{l}\text { Biskut kraker } \\
\text { mil penuh }\end{array}$ & 5 & $3.56 \pm 5.02$ & - & - & - & $1.40 \pm 1.31$ & $4.96 \pm 5.74$ \\
\hline Corn flakes & Emping jagung & 6 & $5.10 \pm 0.90$ & $1.92 \pm 0.47$ & $1.52 \pm 0.43$ & - & $0.05 \pm 0.05$ & $8.58 \pm 1.09$ \\
\hline Muesli & Muesli & 6 & $0.43 \pm 0.43$ & $7.70 \pm 1.77$ & $8.68 \pm 1.76$ & $0.83 \pm 0.83$ & $1.12 \pm 1.12$ & $18.75 \pm 2.05$ \\
\hline Biscuit, milk & Biskut susu & 6 & $17.52 \pm 3.37$ & $0.31 \pm 0.77$ & $0.34 \pm 0.83$ & $0.19 \pm 0.46$ & $1.04 \pm 1.77$ & $19.06 \pm 2.78$ \\
\hline Biscuit, raisin & Biskut kismis & 4 & $11.63 \pm 8.58$ & $4.26 \pm 3.45$ & $5.05 \pm 4.26$ & $3.34 \pm 6.05$ & $0.31 \pm 0.61$ & $24.56 \pm 6.48$ \\
\hline Cookies, butter & Biskut mentega & 6 & $20.63 \pm 2.56$ & $0.22 \pm 0.35$ & $0.21 \pm 0.33$ & $0.66 \pm 1.62$ & - & $21.62 \pm 2.30$ \\
\hline Oatmeal cereal, dry & Bijirin oat, kering & 6 & $1.20 \pm 0.28$ & - & - & - & - & $1.20 \pm 0.28$ \\
\hline Barley flour & Tepung barli & 1 & 3.83 & - & - & - & - & 3.83 \\
\hline Egg noodles & Mee telur & 6 & - & - & - & - & $0.21 \pm 0.51$ & $0.21 \pm 0.51$ \\
\hline Flour, rye & Tepung rye & 4 & $0.71 \pm 0.13$ & - & - & - & - & $0.71 \pm 0.13$ \\
\hline Nestum cereal, dry & Bijirin nestum, kering & 1 & 7.20 & 0.80 & - & - & 3.18 & 11.15 \\
\hline Premix flour & Tepung pracampuran & 6 & $1.44 \pm 3.84$ & - & - & - & - & $1.44 \pm 3.84$ \\
\hline Spaghetti, dry & Spageti, kering & 6 & $0.27 \pm 0.30$ & - & - & - & $2.10 \pm 0.38$ & $2.33 \pm 0.38$ \\
\hline Flour,wheat, self-raising & Tepung naik sendiri & 6 & $0.19 \pm 0.29$ & - & - & - & - & $0.19 \pm 0.29$ \\
\hline Bun, chocolate & Ban coklat & 4 & $6.82 \pm 3.34$ & $4.30 \pm 1.32$ & $4.93 \pm 1.33$ & $0.31 \pm 0.40$ & $1.45 \pm 0.39$ & 17.81 \\
\hline Bun, coconut & Ban kelapa & 6 & $13.59 \pm 2.71$ & $3.96 \pm 0.93$ & $4.54 \pm 1.31$ & - & $1.30 \pm 0.53$ & 23.18 \\
\hline Bun, kaya & Ban kaya & 3 & $15.19 \pm 2.18$ & $3.33 \pm 1.54$ & $3.58 \pm 0.97$ & - & $0.70 \pm 0.42$ & 22.82 \\
\hline Bun, potato & Ban kentang & 6 & $3.12 \pm 1.93$ & $3.50 \pm 0.90$ & $5.03 \pm 1.74$ & $0.35 \pm 0.32$ & $1.33 \pm 0.68$ & 13.55 \\
\hline Bun, red beans & Ban kacang merah & 3 & $12.70 \pm 3.48$ & $2.60 \pm 0.71$ & $3.12 \pm 0.62$ & - & $0.69 \pm 0.46$ & 19.12 \\
\hline
\end{tabular}

(continue on next page) 
Table 1. (Continued)

\begin{tabular}{|c|c|c|c|c|c|c|c|c|}
\hline English name & Malaysian name & $\mathrm{n}$ & Sucrose & Glucose & Fructose & Lactose & Maltose & Total Sugar \\
\hline \multicolumn{9}{|c|}{ Starchy root and tuber products } \\
\hline Breadfruits chips & Kerepek sukun & 4 & $1.94 \pm 0.51$ & - & - & - & - & $1.94 \pm 0.51$ \\
\hline Potato chips, spicy & $\begin{array}{l}\text { Keropok ubi kentang } \\
\text { berperisa rempah pedas }\end{array}$ & 5 & $2.51 \pm 0.83$ & $0.16 \pm 0.37$ & $0.10 \pm 0.21$ & - & - & $2.75 \pm 0.50$ \\
\hline Tapioca chips, spicy & Kerepek ubi kayu pedas & 6 & $7.69 \pm 3.88$ & $2.19 \pm 2.86$ & $2.19 \pm 2.86$ & - & $0.65 \pm 1.01$ & $12.54 \pm 8.39$ \\
\hline $\begin{array}{l}\text { Sweet potato, } \\
\text { red, chips }\end{array}$ & Kerepek ubi keledek & 4 & $4.99 \pm 0.40$ & - & - & - & - & $5.09 \pm 0.56$ \\
\hline $\begin{array}{l}\text { Tapioca chips, } \\
\text { barbeque }\end{array}$ & $\begin{array}{l}\text { Kerepek ubi kayu } \\
\text { perisa barbeque }\end{array}$ & 5 & $10.61 \pm 11.31$ & - & $0.35 \pm 0.33$ & - & - & $10.95 \pm 11.36$ \\
\hline $\begin{array}{l}\text { Tapioca chips, } \\
\text { black pepper }\end{array}$ & $\begin{array}{l}\text { Kerepek ubi kayu } \\
\text { perisa lada hitam }\end{array}$ & 3 & $4.35 \pm 0.79$ & $0.21 \pm 0.36$ & $0.37 \pm 0.34$ & - & - & $4.92 \pm 1.29$ \\
\hline $\begin{array}{l}\text { Tapioca chips, } \\
\text { plain, salted }\end{array}$ & $\begin{array}{l}\text { Kerepek ubi } \\
\text { kayu bergaram }\end{array}$ & 3 & $2.67 \pm 0.55$ & - & $0.19 \pm 0.33$ & - & - & $2.85 \pm 0.89$ \\
\hline $\begin{array}{l}\text { Tapioca chips, } \\
\text { plain, unsalted }\end{array}$ & $\begin{array}{l}\text { Kerepek ubi kayu } \\
\text { tanpa garam }\end{array}$ & 6 & $5.33 \pm 3.55$ & $0.48 \pm 1.17$ & $0.36 \pm 0.38$ & - & - & $7.61 \pm 5.87$ \\
\hline \multicolumn{9}{|l|}{ Legume products } \\
\hline Black eye bean & Kacang mata hitam & 6 & $2.38 \pm 0.43$ & - & - & - & - & $2.38 \pm 0.44$ \\
\hline Carob flour & Tepung kacang kuda & 6 & $2.05 \pm 0.53$ & - & $0.10 \pm 0.25$ & - & - & $2.15 \pm 0.50$ \\
\hline Soya flour & Tepung kacang soya & 6 & $5.57 \pm 2.73$ & - & $0.77 \pm 0.40$ & - & - & $6.33 \pm 3.12$ \\
\hline Soya sauces, sweet & Kicap manis & 6 & $28.67 \pm 15.41$ & $7.25 \pm 2.66$ & $5.00 \pm 1.89$ & - & $1.39 \pm 0.96$ & $42.23 \pm 15.85$ \\
\hline \multicolumn{9}{|l|}{ Nut and seed products } \\
\hline Broad bean & Kacang parang & 6 & $1.37 \pm 0.43$ & - & - & - & - & $1.37 \pm 0.43$ \\
\hline Coconut, shreded & Kelapa parut & 3 & $3.64 \pm 0.29$ & $0.62 \pm 0.06$ & $0.16 \pm 0.28$ & - & - & $4.33 \pm 0.55$ \\
\hline Flaxseed & Biji flaks & 6 & $1.67 \pm 0.17$ & - & - & - & - & $1.69 \pm 0.15$ \\
\hline Ginkgo Nuts & Kacang ginkgo & 6 & $0.85 \pm 0.64$ & $0.51 \pm 0.56$ & $0.32 \pm 0.37$ & - & - & $1.68 \pm 0.70$ \\
\hline Hazelnuts & Kacang hazel & 2 & $1.71 \pm 0.64$ & - & - & - & - & $1.72 \pm 0.64$ \\
\hline Macadamia nuts & Kacang makadamia & 4 & $4.28 \pm 1.02$ & - & - & - & - & $4.28 \pm 1.02$ \\
\hline Peanut, crush & Kacang tumbuk & 6 & $25.92 \pm 13.69$ & $8.11 \pm 10.51$ & $0.77 \pm 0.65$ & - & $5.76 \pm 4.50$ & $40.97 \pm 8.41$ \\
\hline $\begin{array}{l}\text { Peanut/Groundnut, } \\
\text { f lour coated }\end{array}$ & $\begin{array}{l}\text { Kacang tanah } \\
\text { bersalut tepung }\end{array}$ & 3 & $7.79 \pm 2.61$ & - & $0.27 \pm 0.24$ & - & - & $8.06 \pm 2.41$ \\
\hline Pistachio nut & Kacang pistasio & 6 & $5.40 \pm 0.31$ & - & $1.01 \pm 0.78$ & - & - & $6.41 \pm 0.82$ \\
\hline Pumpkin seed & Biji labu & 6 & $1.10 \pm 0.07$ & - & - & - & - & $1.10 \pm 0.05$ \\
\hline \multicolumn{9}{|l|}{ Vegetables } \\
\hline Baby corn & Pucuk jagung & 6 & - & $2.75 \pm 0.17$ & $0.43 \pm 0.28$ & - & - & $3.15 \pm 0.37$ \\
\hline Capsicum, green & Lada bengala hijau & 6 & - & - & $2.76 \pm 0.41$ & - & - & $2.76 \pm 0.41$ \\
\hline Capsicum, red & Lada bengala merah & 6 & & $3.23 \pm 0.40$ & $2.04 \pm 1.03$ & - & - & $4.83 \pm 1.30$ \\
\hline Capsicum, yellow & Lada bengala kuning & 6 & - & $2.74 \pm 0.64$ & - & - & - & $2.74 \pm 0.64$ \\
\hline \multicolumn{9}{|l|}{ Fruits } \\
\hline Dragon fruit, red & Buah naga isi merah & 3 & - & 5.21 & 1.53 & - & 1.64 & 8.40 \\
\hline Dragon fruit, white & Buah naga isi putih & 3 & - & 5.48 & 2.08 & - & 0.94 & 8.50 \\
\hline Honeydew & Tembikai susu & 4 & - & $1.31 \pm 0.23$ & $1.61 \pm 0.36$ & - & - & $2.92 \pm 0.59$ \\
\hline Pomegranate & Delima & 3 & & 5.42 & 5.28 & - & - & 10.70 \\
\hline Watermelon, yellow & Tembikai kuning & 2 & - & 1.82 & 2.50 & - & - & 4.30 \\
\hline \multicolumn{9}{|c|}{ Sugar and syrup products } \\
\hline Cordial root beer & Kordial rut bir & 2 & $3.64 \pm 5.14$ & $5.79 \pm 5.35$ & $5.59 \pm 5.31$ & - & - & $15.00 \pm 5.52$ \\
\hline Cordial, grape & Kordial anggur & 6 & $0.59 \pm 0.97$ & $16.46 \pm 12.15$ & $17.13 \pm 13.71$ & - & - & $34.9 \pm 26.22$ \\
\hline Cordial, guava & Kordial jambu batu & 4 & $16.47 \pm 19.18$ & $24.30 \pm 12.12$ & $23.86 \pm 11.70$ & - & - & $64.63 \pm 7.03$ \\
\hline Cordial, kiwi & Kordial kiwi & 4 & - & $10.40 \pm 13.80$ & $9.96 \pm 13.05$ & - & - & $20.40 \pm 26.88$ \\
\hline Cordial, lime & Kordial limau nipis & 4 & - & $15.97 \pm 8.94$ & $15.20 \pm 8.47$ & - & - & $31.22 \pm 17.41$ \\
\hline Cordial, mango & Kordial mangga & 6 & $2.50 \pm 3.37$ & $20.78 \pm 10.87$ & $20.18 \pm 10.58$ & - & - & $43.46 \pm 22.08$ \\
\hline
\end{tabular}


Table 1. (Continued)

\begin{tabular}{|c|c|c|c|c|c|c|c|c|}
\hline English name & Malaysian name & $\mathrm{n}$ & Sucrose & Glucose & Fructose & Lactose & Maltose & Total Sugar \\
\hline \multicolumn{9}{|l|}{ Sugar and syrup products } \\
\hline Cordial, orange & Kordial oren & 6 & $1.27 \pm 2.85$ & $20.41 \pm 9.72$ & $19.72 \pm 9.25$ & - & - & $41.41 \pm 18.89$ \\
\hline Cordial, pineapple & Kordial nenas & 4 & $2.90 \pm 3.65$ & $32.64 \pm 5.13$ & $29.97 \pm 5.45$ & - & - & $65.52 \pm 13.53$ \\
\hline Cordial, roselle & Kordial roselle & 6 & $3.63 \pm 8.89$ & $24.10 \pm 9.88$ & $23.30 \pm 9.50$ & - & - & $51.03 \pm 18.50$ \\
\hline Cordial, sarsi & Kordial sarsi & 6 & $6.25 \pm 5.40$ & $13.43 \pm 10.21$ & $13.23 \pm 10.09$ & - & - & $32.91 \pm 22.12$ \\
\hline Cordial, soursop & Kordial durian belanda & 6 & $1.99 \pm 4.09$ & $21.87 \pm 13.79$ & $21.52 \pm 13.63$ & - & - & $45.39 \pm 29.61$ \\
\hline Jam, apricot & Jem aprikot & 6 & $8.47 \pm 4.60$ & $23.33 \pm 1.78$ & $19.85 \pm 1.94$ & - & $2.00 \pm 1.34$ & $53.65 \pm 1.96$ \\
\hline Jam, blueberry & Jem blueberi & 6 & $5.08 \pm 4.32$ & $23.73 \pm 1.70$ & $21.72 \pm 1.65$ & - & $4.27 \pm 2.34$ & $54.78 \pm 2.83$ \\
\hline Jam, grape & Jem anggur & 6 & $1.02 \pm 1.02$ & $26.20 \pm 4.49$ & $26.00 \pm 4.69$ & - & - & $52.43 \pm 9.48$ \\
\hline Jam, strawberry & Jem strawberi & 6 & $9.78 \pm 4.72$ & $17.42 \pm 3.11$ & $15.77 \pm 2.80$ & - & $9.68 \pm 5.53$ & $52.67 \pm 1.94$ \\
\hline \multicolumn{9}{|l|}{ Meat products } \\
\hline Sausage, beef & Sosej daging ayam & 6 & - & - & - & - & 0.255 & 0.255 \\
\hline Sausage, chicken & Sosej daging ayam & 6 & $0.32 \pm 0.07$ & $0.68 \pm 0.71$ & 0.20 & - & - & $0.543 \pm 0.60$ \\
\hline \multicolumn{9}{|l|}{ Oils and fats } \\
\hline Mayonnaise & Mayonis & 6 & $5.48 \pm 1.23$ & $1.71 \pm 0.31$ & $1.40 \pm 0.2$ & - & $0.18 \pm 0.18$ & $8.59 \pm 1.20$ \\
\hline Sauce, thousand island & Sos thousand island & 6 & $5.35 \pm 1.55$ & $4.28 \pm 1.03$ & $5.83 \pm 2.31$ & - & - & $15.47 \pm 2.40$ \\
\hline
\end{tabular}

\section{Conclusions}

This data showed that most cereal products contained sucrose, glucose, fructose, lactose and maltose; most starchy roots and tubers products contained sucrose, glucose and fructose; all legume products, nuts and seed products contained sucrose; all five fruits contained glucose and fructose and syrups contained the highest sucrose compared to all other food groups. Overall, sugars and syrups products contained the most total sugar and vegetables the least. These data provide information on the sugar content of various food products in Malaysia that will help to assist the public in making healthy food choices.

\section{Acknowledgements}

The authors thank the Director General of Health Malaysia and the Director of Institute for Medical research (IMR) for permission to publish this article. We also thank the staff of the Nutrition Unit, Institute for Medical for their support in this study. This study was funded by the Ministry of Health Grant.

\section{References}

Bray, G. A. (2013). Energy and fructose from beverages sweetened with sugar or high-fructose corn syrup pose a health risk for some people. Advances in Nutrition, 4, 220-225. https://doi.org/10.3945/an.112.002816

Kahn, R., \& Sievenpiper, J. L. (2014). Dietary sugar and body weight: have we reached a crisis in the epidemic of obesity and diabetes? We have, but the pox on sugar is overwrought and overworked. Diabetes Care, 37, 957-962. https://doi.org/10.2337/dc13-2506

Ministry of Health Malaysia. (2008). Malaysian adult nutrition survey 2003. Habitual intake of adults aged 18 to 59 years. Putrajaya, Malaysia: Nutrition Section, Family Health Development Division, Ministry of Health Malaysia.

Morenga, L. T., Mallard, S., \& Mann, J. (2013). Dietary sugars and body weight: systematic review and meta-analyses of randomised controlled trials and cohort studies. BMJ. 346, 7492. https://doi.org/10.1136/bmj.e7492

National Technical Group of Malaysian Food Composition Database 2011. (2011). Protocol for Sampling and Method of Analysis for Malaysian Food Composition Database. Kuala Lumpur: National Technical Group of Malaysian Food Composition Database.

Norhayati, M. K., Mohd Fairulnizal, M. N., Zaiton, A., Wan Syuriahti, W. Z., Rusidah, S., Aswir, A. R., ... Vimala, B. (2015). Nutritional composition of selected commercial biscuits in Malaysia. Sains Malaysiana, 44(4), 581-591. https://doi.org/10.17576/jsm-2015-4404-13

Sanchez-Castillo, C. P., Dewey, P. J. S., Lara, J. J., Henderson, D. L., Lourdes Solano, M. D., \& James, W. P. T. (2000). The starch and sugar content of some Mexican cereals, cereal products, pulses, snack foods, fruits and vegetables. Journal of Food Composition and Analysis, 13, 157-170.

https://doi.org/10.1006/jfca.1999.0866 
Sigman-Grant, M., \& Morita, J. (2003). Defining and interpreting intakes of sugars. The American Journal of Clinical Nutrition, 78, 815-826. https://doi.org/10.1093/ajcn/78.4.815S

Siong, T. E, Noor, M. I., Azudin, M. N., \& Idris, K. (1997). Nutrient composition of Malaysian foods $4^{\text {th }}$ ed. Kuala Lumpur: Malaysian Food composition Database Programme c/o Institute for Medical Research.

Tappy, L., \& Le, K. A. (2010). Metabolic effects of fructose and the worldwide increase in obesity. Physiological Reviews, 90, 23-46. https://doi.org/10.1152/physrev.00019.2009

Van Horn, L., Johnson, R. K., Flickinger, B. D., Vafiadis, D. K., \& Yin-Piazza, S. (2010). Translation and implementation of added sugars consumption recommendations: a conference report from the American Heart Association Added Sugars Conference 2010. Circulation, 122, 2470-2490.

https://doi.org/10.1161/CIR.0b013e3181ffdcb0

\section{Copyrights}

Copyright for this article is retained by the author(s), with first publication rights granted to the journal.

This is an open-access article distributed under the terms and conditions of the Creative Commons Attribution license (http://creativecommons.org/licenses/by/4.0/). 